\title{
Thermodynamic and Diffusion Simulation: A Power Tool for Materials Design
}

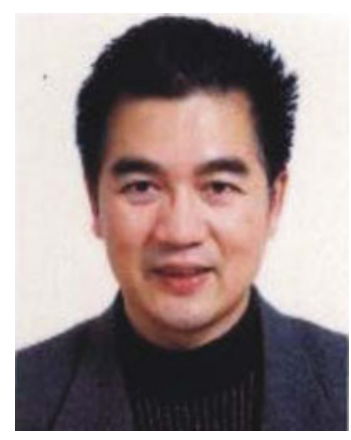

It was in September 1984 that I came to know of the Journal of Phase Equilibria and Diffusion (formerly Bulletin of Alloy Phase Diagrams). At that time, I was one of the postgraduates in Prof. Z. P. Jin's group. Like many of my colleagues in Changsha, I passed competitive examinations for many courses including thermodynamics (phase diagram) and diffusion courses with reasonable grades, but had no clear idea what we can do with the knowledge of both thermodynamics and diffusivity from the technological point of view.

My subsequent research investigation from 1984 to 2002 was mainly about measurement of phase diagram, CALPHAD approach, diffusion modeling, and a fundamental modeling of solidification and welding processes. During this said period, I had the chance to do research work as a $\mathrm{Ph} . \mathrm{D}$ candidate or Postdoc in six groups: Prof. Z. P. Jin's group (Central South University of China), Prof. M. Yoshimura's group (Tokyo Institute of Technology), Prof. N. Clavaguera's group (University of Barcelona), Prof. R. Schmid-Fetzer's group (TU Clausthal), Prof. J. C. Schuster's group (University of Vienna), and Prof. Y. A. Chang's group (University of Wisconsin-Madison). The message I received from the above professors and some other scientists in our field led me to conclude that I am a good researcher probably due to my publications in peer-review international journals. Through the research work in these groups with somewhat different backgrounds, I had a good command of theories in both thermodynamics and diffusion, which are perhaps the most important two aspects of materials science to the best of my knowledge. To some extent, I began to realize the power of both thermodynamics and diffusion for understanding the processes of materials, such as solidification and welding. During this period, it could be said that life was easy for me since I could publish many articles using well-established or slightly modified computational and experimental tools by means of the available research projects of the professors.

The situation changed when I was appointed as Professor at Central South University of China in March 2003. One of a few important duties for me as a professor is to submit research proposals which are used to train students who can then do scientific research work. With more and more students in my group, I had the severe pressure to obtain more projects. Generally speaking, nowadays many governmental financial agencies worldwide, such as the National Natural Science Foundation of China and German Science Foundation, are showing less interest to support fundamental projects associated with thermodynamics or phase diagram or diffusivity. Owing to the financial difficulty, many laboratories in these fields are closed down. For example, only a few places including Illinois Institute of Technology (Prof. P. Nash) and University of Vienna (Prof. H. Ipser) are still left worldwide with the competence to measure the enthalpy of formation for compounds. This enthalpy value is the dominant term of the Gibbs free energy. It is unlikely that governmental financial agencies could support more fundamental projects about thermodynamics and diffusivity in the near future. As thermodynamic and diffusion modeling is an important tool for materials design, our work in this field should continue. One way to overcome the present financial difficulty in the fields of thermodynamics and diffusion modeling is to perform a joint research work with engineers in industry. Through this cooperative work, students can not only deepen their understanding on theory but also show more interest on both thermodynamics and diffusion modeling since they see the real outcome of the modeling in industry. In the following, I will briefly summarize our latest attempt to merge the thermodynamic and diffusion simulation with the production of gradient cemented carbides. And, I will be happy to share the experience with readers for the industrial application of thermodynamics and diffusion simulation.

Cemented carbides consist of hard WC grains embedded in a more ductile binder phase (Co). Often, other hard-phase grains are also present in the form of carbides or carbo-nitrides with a cubic structure. The later hard-phase contains Ti, W, Ta, Nb, etc. An important application for cemented carbides, often in the form of tool inserts, is cutting of materials. To increase the lifespan of the cemented carbide inserts during the cutting, the wear surface is usually coated with a thin layer of a hard material, such as $\mathrm{TiC}, \mathrm{TiN},(\mathrm{Ti}, \mathrm{Al}) \mathrm{N},(\mathrm{Ti}, \mathrm{Al}, \mathrm{Zr}) \mathrm{N}$, or multilayers of such hard materials. These layers are usually grown by means of chemical vapor deposition (CVD) at about $1000{ }^{\circ} \mathrm{C}$. Owing to the difference in thermal expansion coefficients between coating and insert, cracks are formed in the coating during cooling after CVD process. To prevent these cracks from propagating into the bulk and causing failure, a tough surface zone is created before the coating. This zone is enriched in binder phase and depleted in cubic carbides. The production of this kind of cemented carbide is usually divided into two steps: a presintering, and a gradient sintering. The pre-sintering, which also includes a dewaxing step to remove the press lubricant and a de-oxidation step, is conducted in solid state to obtain a dense body without the formation of compositional gradients. The second sintering, the gradient sintering, is performed in liquid state with a holding time of a few hours in a nitrogen-free atmosphere or an atmosphere with several dozen mbar partial pressure for nitrogen. By gradient sintering, an outward diffusion of nitrogen will occur. Titanium-rich cubic carbides dissolve, and a strong thermodynamic coupling between nitrogen and titanium leads to an inward diffusion of titanium. Consequently, a surface zone, depleted in cubic carbides and enriched in binder phase, is created. The distribution of WC, binder phase (Co), and fcc carbides or carbo-nitrides from the surface to the 
bulk of the insert as well as the control of the tough surface zone are the key issues for a successful production of the gradient cemented carbides.

In June 2009, Zhuzhou Cemented Carbide Cutting Tools Limited Company, which is the largest cemented carbide company in China, became interested in the manufacture of the $\mathrm{WC}+\mathrm{Co}+\mathrm{Ti}(\mathrm{C}, \mathrm{N})$ gradient cemented carbides. In order to control the microstructure, in particular the thickness of the tough surface zone, seven variables (contents of 4 components, temperature, time, and atmosphere for gradient sintering) must be selected preciously during the gradient sintering of this five-component cemented carbide. Without thermodynamic calculations and diffusion modeling, it would be extremely difficult for engineers in this company to find an optimal combination of these seven variables experimentally. For this purpose, we have established the thermodynamic and atomic mobility databases for multicomponent cemented carbides with the grant of several research projects from this company. Armed with the established databases, DICTRA simulation can well predict the measured concentration profiles for various components and the volume fractions of various phases as a function of alloy composition as well as temperature, time, and atmosphere used for gradient sintering. The good agreement between the calculated and experimental values highlights the power of thermodynamic calculation and diffusion modeling for guiding the production of the gradient cemented carbides highly efficiently. More detail about our research in computer simulation for the formation of gradient cemented carbides and its experimental verification can be found elsewhere [W.B. Zhang et al., Acta Mater. Sinica, 2011, 47(10), p 1307-1314].

It is worth mentioning that similar simulations on the formation of gradient cemented carbides were published by Ekroth et al. [M. Ekroth et al., Acta Mater. 2000, 48(9), p 2177-2185]. However, the thermodynamic and atomic mobility databases used by them are not available commercially. In the future, it is of interest to simulate $2 \mathrm{D}$ or even $3 \mathrm{D}$ microstructure during gradient sintering by means of phase field method. The simulated microstructure can then be integrated with a hardness model to predict the variation of hardness from the surface to the bulk of the gradient cemented carbide. It is highly expected that the integration of these simulations with decisive experiment will reduce the cycle for the development of new gradient cemented carbides significantly. I am convinced that more and more engineers in industry will realize the importance of thermodynamic calculations and diffusion modeling if we could demonstrate successful applications of these simulations in practical productions of materials. As a consequence, many research groups, like my research group, could survive through the research projects not only from governmental financial agencies but also from companies. 